
در شرايط آب و هوايى استان خوز فئستان

\author{
كاوه ليموجى '، مهرداد يارنيا'، عطا اله سيادت"، ورهرام رشيدى و و عبدالعلى تيلانى
}

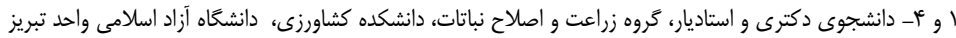

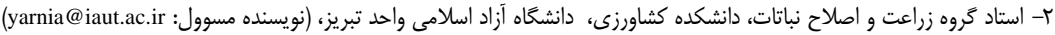

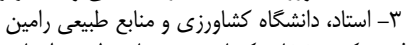
هـ استاديار، عضو هيأت علمى بخش اصلاح و تهيه نهال و بذر، مركز تحقيقات كشاورزى و منابع طبيعى استان خوزستان، سازمان تارئ تحقيقات، آموزش و ترويج كشاورزىى، اهواز تاريخ يذيرش:

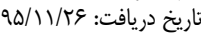

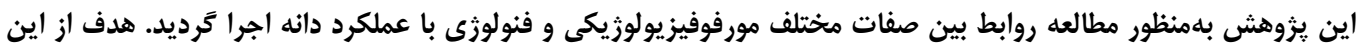

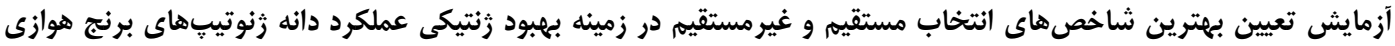

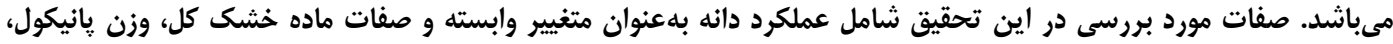

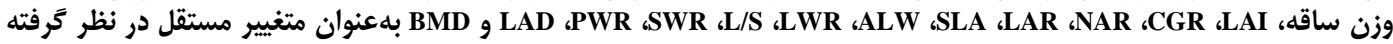

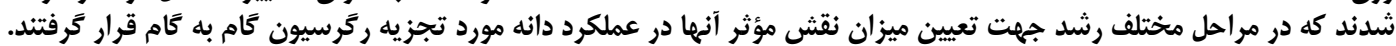

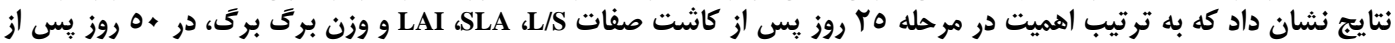

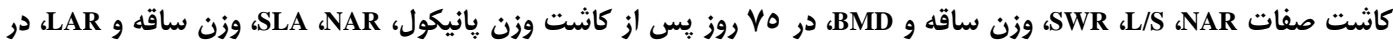

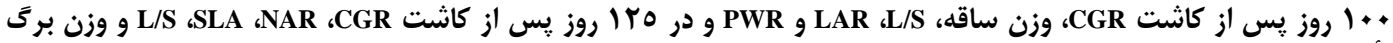

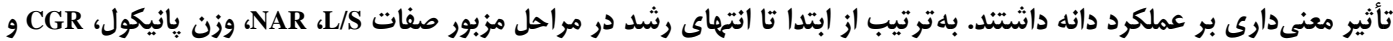

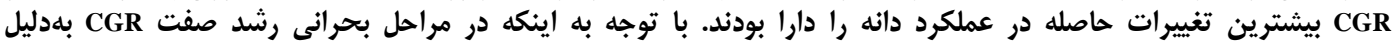

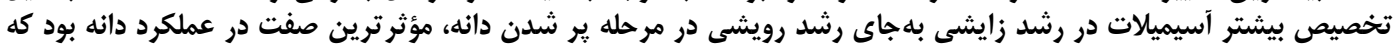

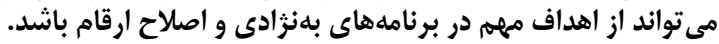

وازمهاى كليدى: مراحل رشد، فيزيولوزى، مورفولوزى، CGR، رَّرسيون كامبهَام

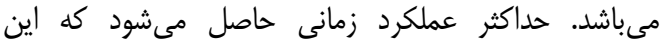

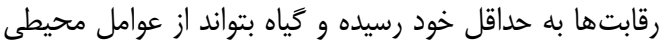

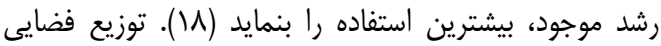

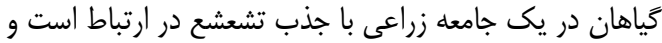

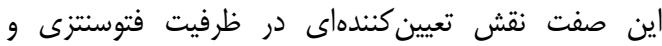

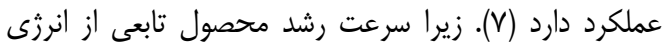

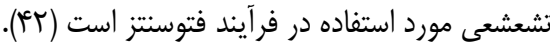

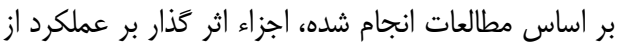

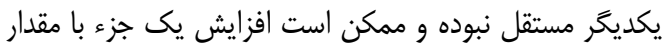

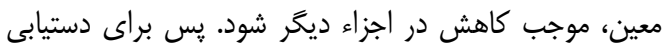

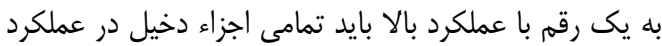

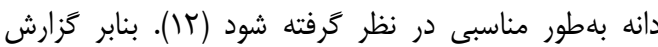

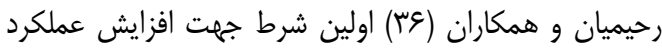

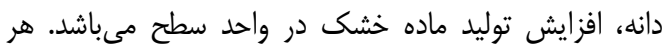

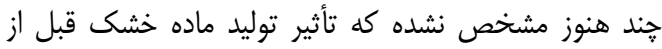

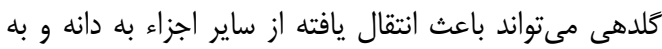

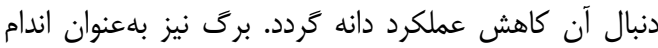

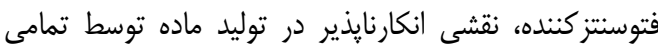

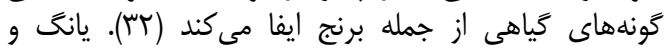

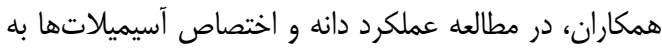

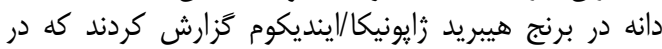

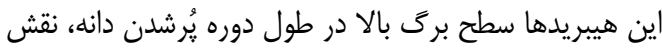

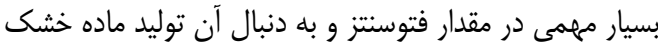

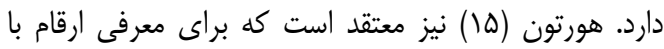

مقدمه

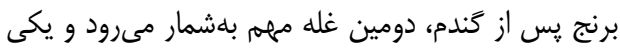

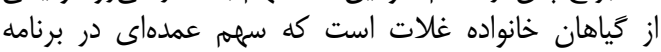

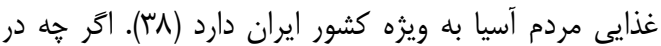

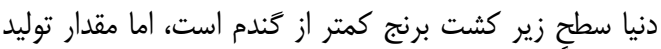

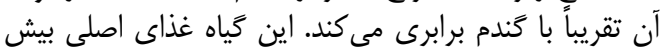

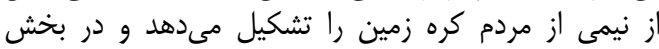

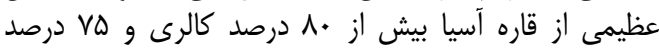

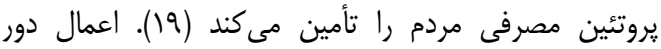

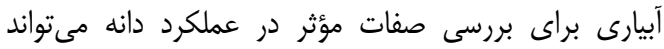

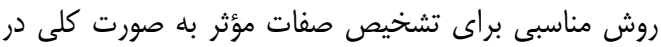

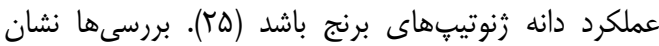

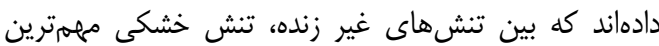

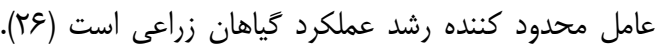

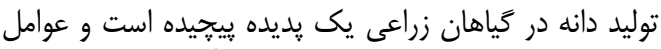

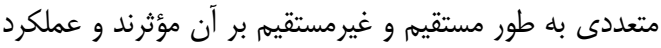

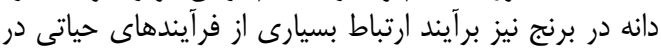

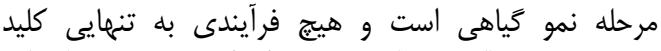

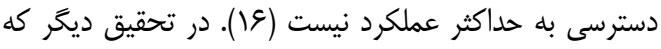

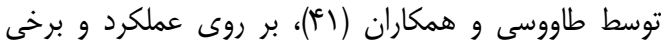

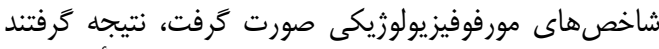

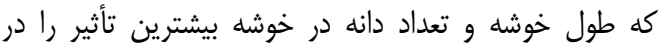

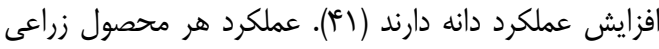
حاصل رقابت برون و درون بوتهاى بران براى عوامل محيطى رشد رشد 
وزن مخصوص برى (SLW) و تجمع ماده خشك (DMA) همبستخى مثبتى داشت.

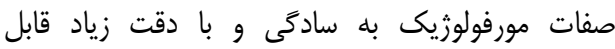

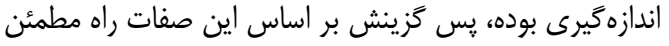

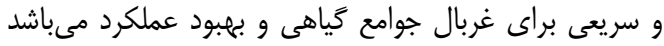

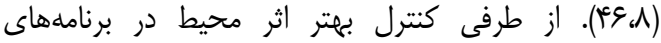

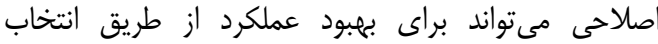

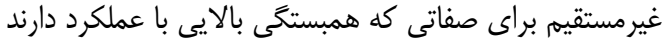

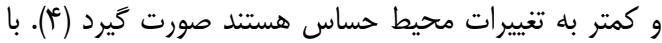

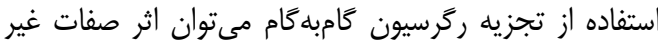

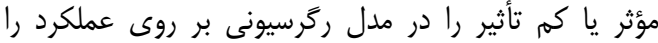

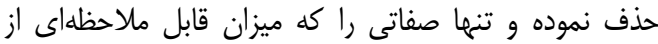

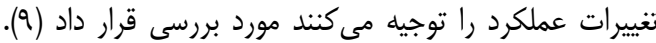

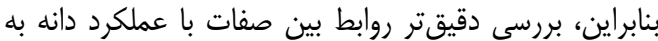

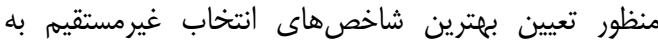

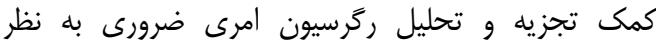

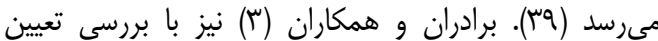
صفات مطلوب و تأثير گذار بر روى عملكرد كلزا كزارش

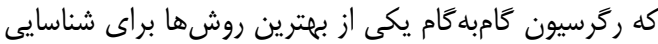

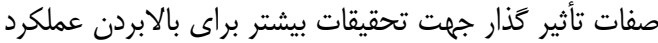

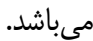

هدف از اين تحقيق، بررسى روابط بين صفات مختلف با

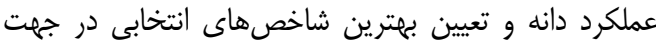

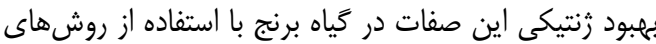

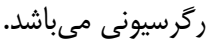

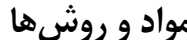

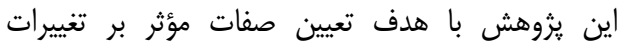

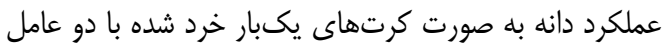

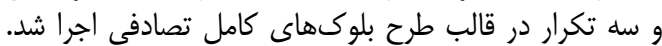

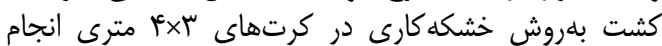

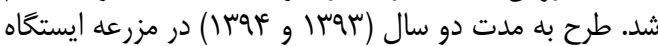

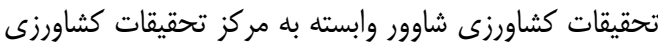

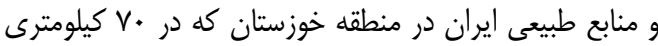

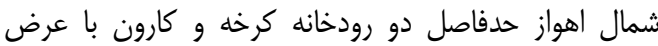

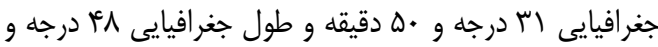

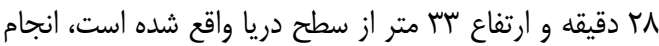

مشخصات فيزيكى -شيميايى خاك مزرعه دره در جدول

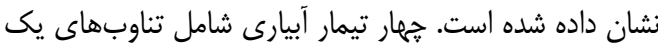

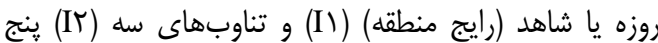

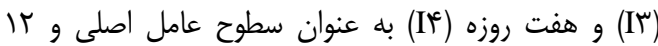

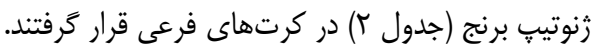

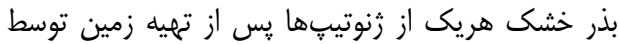

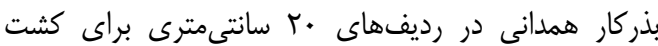

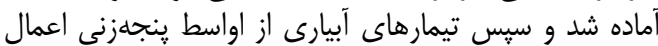

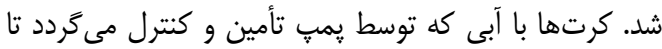

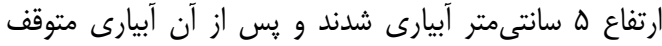

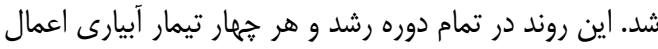

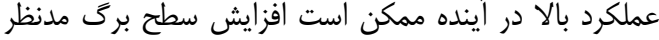

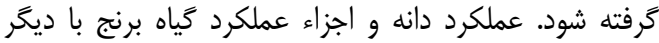

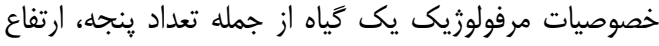

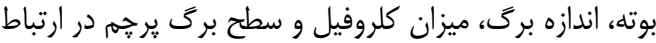

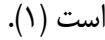

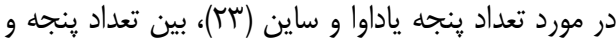

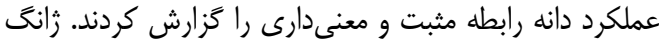

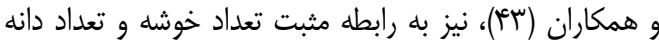

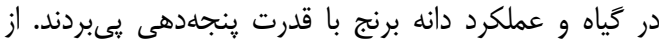

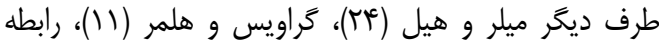

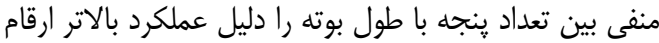

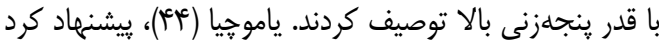

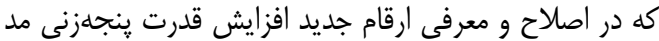

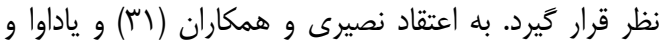
ساين (ساء)، ارتفاع بوته نيز از صفات مؤيرى

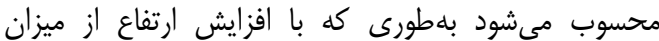

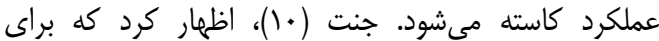

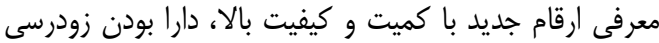

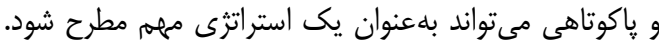

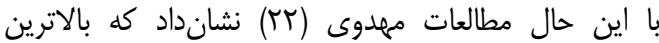

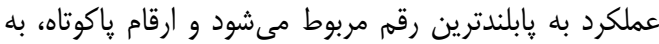

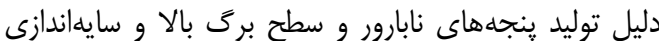
بيشتر، دجار كاهش فتوسنتز و توليد دانه مى شى نئلد.

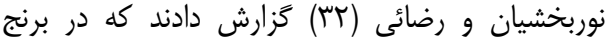
سرعت رشد محصول (CGR) و سرعت رشد شئن نسبى (RGR)

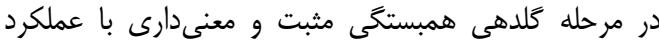

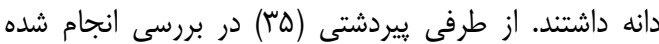

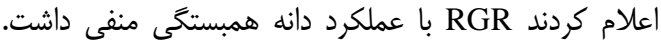

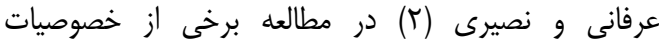

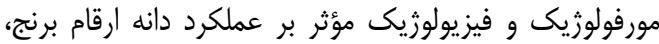

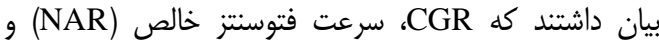
شاخص سطح برى (LAI) در ارقام اصلاح شده در فر تمام

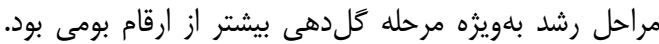

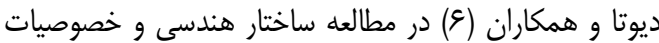

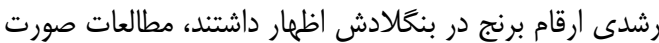

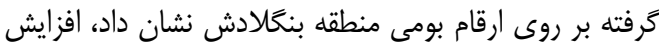

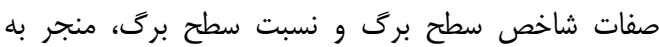

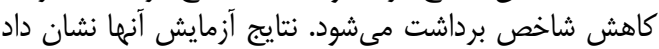

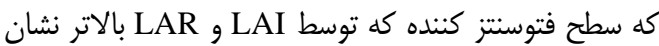

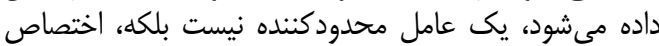

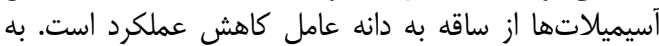

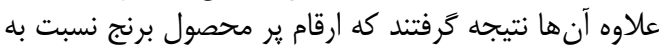

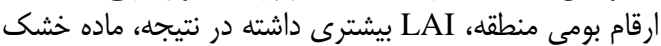

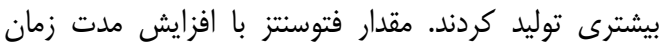

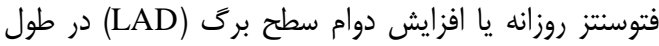

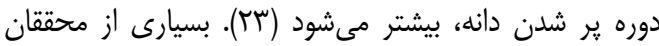

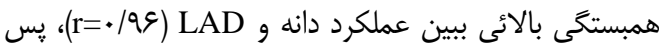

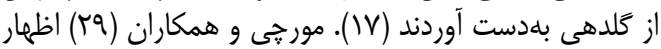
كردند كه در واريتهاى ير محصول برنج، NAR با NGR، 


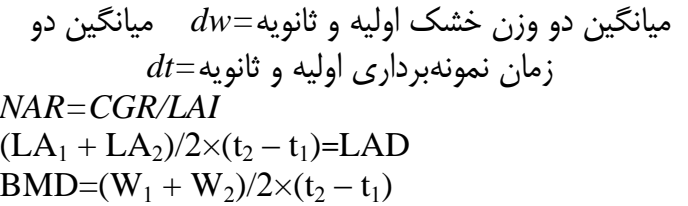

تغييرات سطح برى در دو فاصله زمانى تغييرات وزن برك در دو فاصله زمانى فواصل زمانى نمونه بردارى شده دواصل

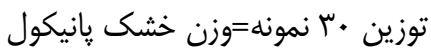
وزن خشك پانيكول در واحد سطح / وزن خشك كل درل در واحد

$$
\begin{aligned}
& \text { SLA }=\frac{\left(\frac{L A 1}{L W 1}+\frac{L A 2}{L W 2}\right)}{2} \\
& L A R=\frac{\left(\frac{L A 1}{W 1}+\frac{L A 2}{W 2}\right)}{2} \\
& L / S=\frac{L W}{S W}
\end{aligned}
$$

سطح= PWR

LA1 و LA2 ميانكين سطح برك در دو فاصله زمانى داصن

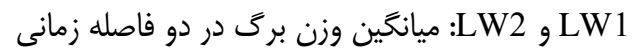

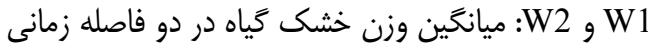

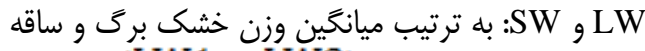

$$
\begin{aligned}
& \text { SLW }=\frac{\left(\frac{L W 1}{L A 1}+\frac{L W 2}{L A 2}\right)}{2{ }^{2}} \\
& \text { SWR }=\frac{\left(\frac{L W 1}{W 1}+\frac{L W 2}{W 2}\right)}{2} \\
& \text { SWR }=\frac{\left(\frac{S W 1}{W 1}+\frac{S W 2}{W 2}\right)}{2}
\end{aligned}
$$

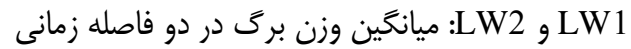

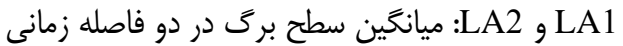

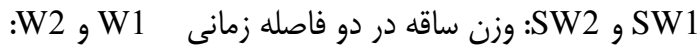
وزن خشى كل كياه در دو فاصله زمانى $R G R=\frac{\operatorname{Ln} W 2-\operatorname{Ln} W 1}{T 2-T 1}$

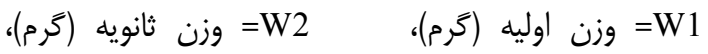

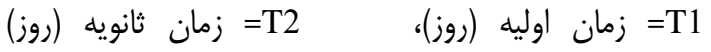
RGR
شد. براى جلوكيرى از نفوذ آب به كرتهاى مجاور تمام

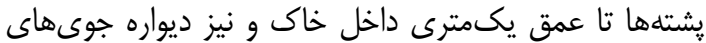

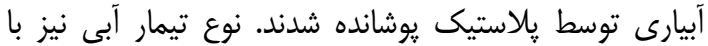

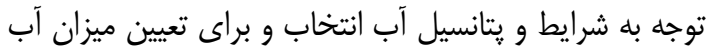

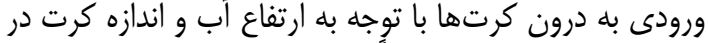

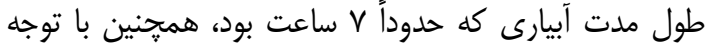

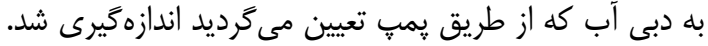

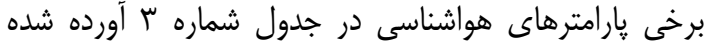

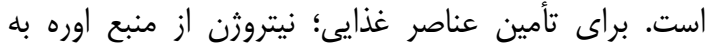

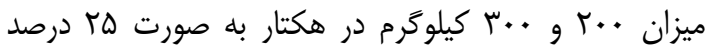

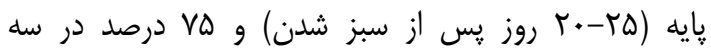

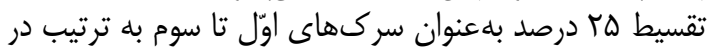

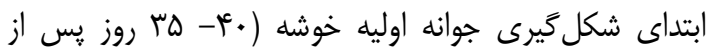

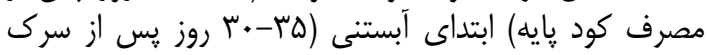

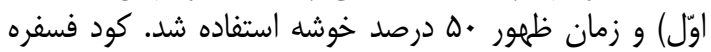

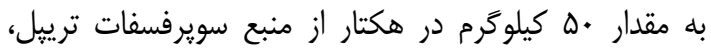

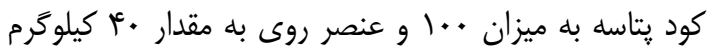

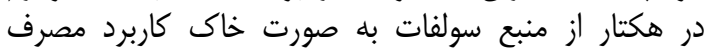

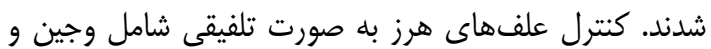

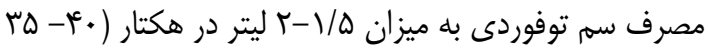
روز يس از سبز شدن) انجام كرديد

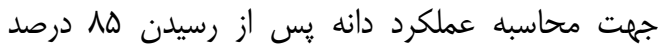

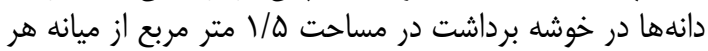

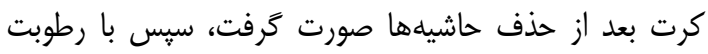

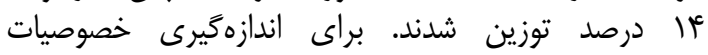

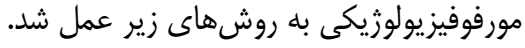

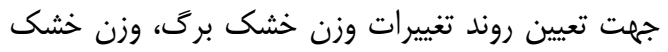

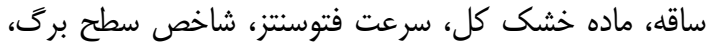

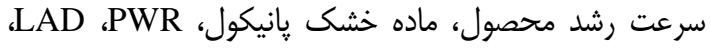
RGR و SLW ،WR ، SWR ، L/S ، LAR ، SLA ، BMD

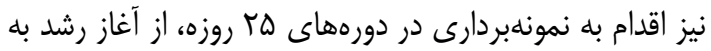

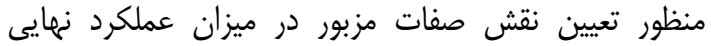

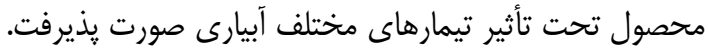

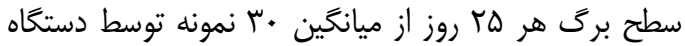

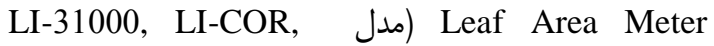
(Lincoln, NE

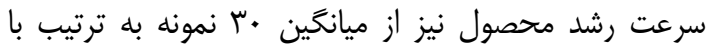
$L A I=L A / S A$ استفاده از روابط زير محاسبه كرديد.

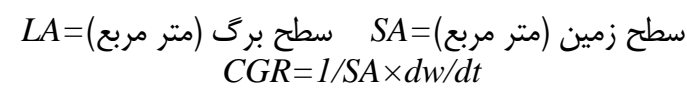


Table 1. Soil characteristics of the research farm

جدول (- مشخصات خاك مزرعه تحقيقاتى

\begin{tabular}{|c|c|c|c|c|c|c|c|c|c|c|}
\hline \multirow[t]{2}{*}{ بافت خاى } & \multicolumn{4}{|c|}{$\begin{array}{c}\text { عناصر ريز مغذى } \\
\text { (ppm) }\end{array}$} & \multirow[t]{2}{*}{$\underset{(\mathrm{ppm})}{\mathrm{K}}$} & \multirow{2}{*}{$\underset{(\mathrm{ppm})}{\mathrm{P}}$} & \multirow{2}{*}{$\underset{(\mathrm{ppm})}{\mathrm{N}}$} & \multirow[t]{2}{*}{$\mathrm{EC}$} & \multirow[t]{2}{*}{$\mathrm{pH}$} & \multirow{2}{*}{$\begin{array}{c}\text { عمق خاك } \\
\text { (cm) }\end{array}$} \\
\hline & $\mathrm{Fe}$ & $\mathrm{Mn}$ & $\mathrm{Zn}$ & $\mathrm{Cu}$ & & & & & & \\
\hline لومى-رسى & $1 Q / / r$ & $\Gamma / \Delta$ & $T / \Delta$ & $r / l$ & THI & 11 & .1 .9 & $\Gamma / \Delta$ & $V / \Gamma$ & • \\
\hline
\end{tabular}

Table 2. Some features of used pedigree genotypes in the study

جدول r- برخى ويزَى ها و شجره زنوتيبهاى مورد استفاده در تحقيق

\begin{tabular}{|c|c|c|c|c|}
\hline تحمل به خشكى & منشاء & تلاقى & رئوتيبٍ & \\
\hline 1 & هند & C 22/KALAKERI & VANDANA & $\mathrm{V}_{1}$ \\
\hline 1 & 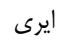 & VANDANA/IR 65 & IR 78908-193-B-3-B & $\mathrm{V}_{2}$ \\
\hline 1 & 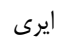 & IR 78908-44/IR 78908-86 & IR 81429-B-31 & $\mathrm{V}_{3}$ \\
\hline r & 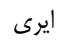 & PSB RC 9/IR 64 & IR 78875-176-B-1-B & $\mathrm{V}_{4}$ \\
\hline a & 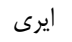 & VANDANA/WAYRAREM & IR 79971-B-202-2-4 & $\mathrm{V}_{5}$ \\
\hline $\mathrm{v}$ & 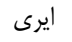 & PSB RC 9/AUS 257 & IR 80508-B-194-4-B & $\mathrm{V}_{6}$ \\
\hline$\Delta$ & 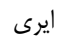 & PSB RC 9/AUS 257 & IR 80508-B-194-3-B & $\mathrm{V}_{7}$ \\
\hline a & 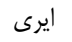 & IR 55419-04/IR 64 & IR 79907-B-493-3-1 & $\mathrm{V}_{8}$ \\
\hline a & 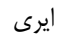 & NSIC RC 140/IR 74371-3-1-1 & IR 81025-B-347-3 & $\mathrm{V}_{9}$ \\
\hline r & 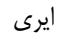 & NSIC RC 140/IR 74371-3-1-1 & IR $81025-B-327-3$ & $\mathrm{~V}_{10}$ \\
\hline r & ايران & SANG TARAM/AMOL $_{3}$ & 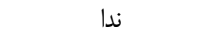 & $\mathrm{V}_{11}$ \\
\hline 9 & ايران & - & 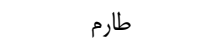 & $\mathrm{V}_{12}$ \\
\hline
\end{tabular}

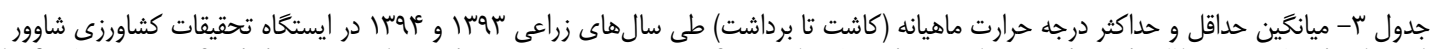
Table 3. Average of minimum and maximum temperature of months (sowing to harvesting) in Shavoor Agricultural Research Station for two years (2014 and 2015)

\begin{tabular}{|c|c|c|c|c|}
\hline \multicolumn{2}{|c|}{$(1) q q)$} & \multicolumn{2}{|c|}{$\left.\left.(1)^{\prime}\right)^{\prime}\right)$} & \multirow[t]{2}{*}{ ماه } \\
\hline (درجه سانتى مرادين حداد) & (درجه سانتى حدراد) & (درجانگين سداكتى مادر) & (درجه سانتى حدراد) & \\
\hline$f a / V$ & rV/A & $ז \& / V$ & $r V / \Lambda$ & تير \\
\hline$F V / \Delta$ & $r q / 1$ & $\leftarrow \& / \Delta$ & $r V / \Lambda$ & مرداد \\
\hline$r q / \Delta$ & $r T / T$ & ب & KI & مهر \\
\hline$r V / \Lambda$ & $1 Q / \Lambda$ & rq & $1 \pi / V$ & آبان \\
\hline$F / / M$ & MF/AT & 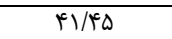 & & ميانخين \\
\hline
\end{tabular}

مىتواند بهدليل افزايش تنفس بر اثر سايهاندازى و همجنين

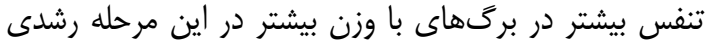

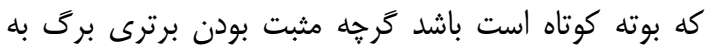

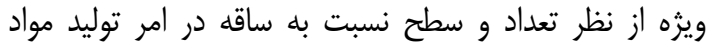

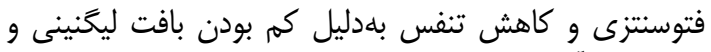

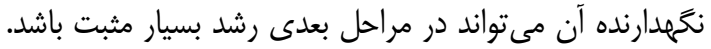

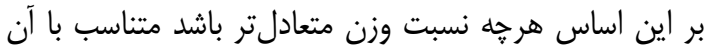

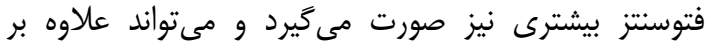

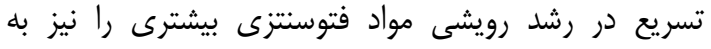

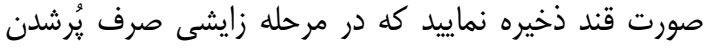

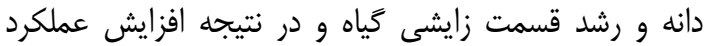

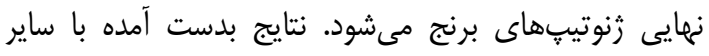

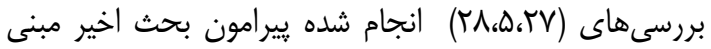

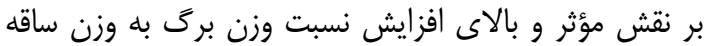
همخوانى دارد.

\section{نتايج و بحث}

هr روز پس از كاشت: نتايج تجزيه رگرسيون گام به گام

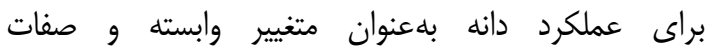

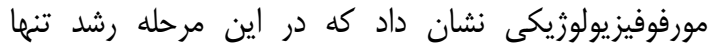

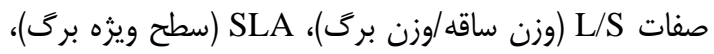
LAI

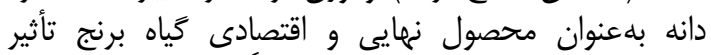

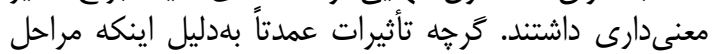

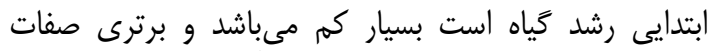

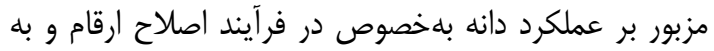

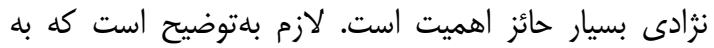

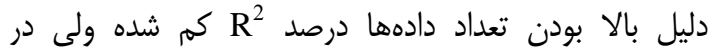

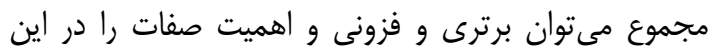

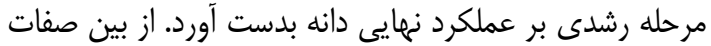

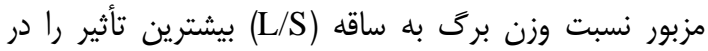

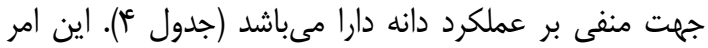


جدول أ- نتايج تجزيه ركر سيون كام به كام عملكرد دانه و صفات مورفوفيزيولوزيكى در فا روز بِ إز كاشت Table 4. Stepwise regression analysis of grain yield and morpho-physiological characteristics after 25 days from planting

\begin{tabular}{|c|c|c|}
\hline 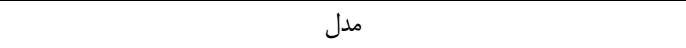 & Partial R2 & Model R2 \\
\hline $\mathrm{Y}=r \varepsilon q \mathrm{~V} / 11 \cdot-119 / \wedge \varepsilon \mathrm{L} / \mathrm{S}$ & .1 .9 & .1 .9 \\
\hline 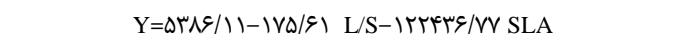 & $\cdot / \cdot 9+\cdot / \cdot r$ &.$/ 14$ \\
\hline 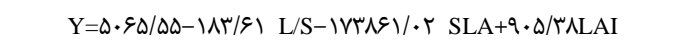 & $\cdot / \cdot 9+\cdot / \cdot r+\cdot / \cdot 1$ &.$/ 1 F$ \\
\hline 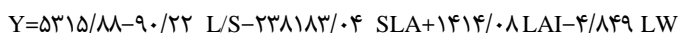 & $\cdot 1 \cdot 9+\cdot 1 \cdot 1+\cdot \cdot \cdot \cdot 1+\cdot / \cdot 1$ &.$/ 10$ \\
\hline
\end{tabular}

L و LAI SLA ،L/S

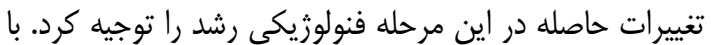

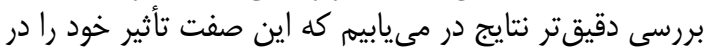

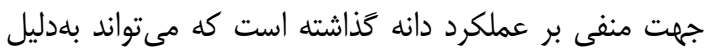

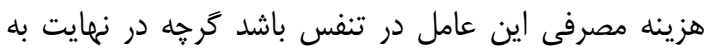

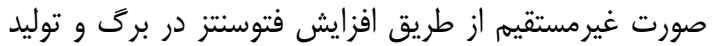

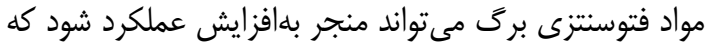

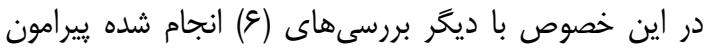
بحث اخير مطابقت دارد.

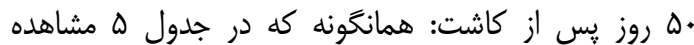

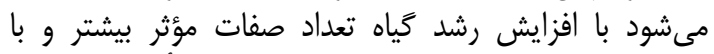

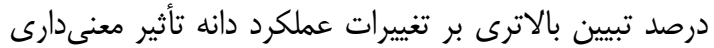

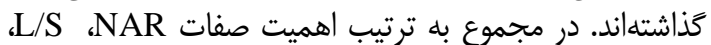

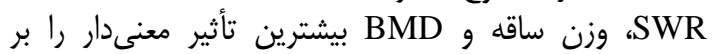

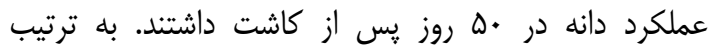

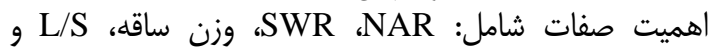

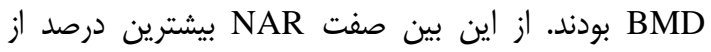

جدول ه- نتايج تجزيه رگرسيون گام به گام عملكرد دانه و صفات مورفوفيزيولوزيكى در •ه روز يس از كاشت Table 5. Stepwise regression analysis of grain yield and morpho-physiological characteristics after 50 days from planting

\begin{tabular}{|c|c|c|}
\hline مدل & Partial $R^{2}$ & Model $R^{2}$ \\
\hline 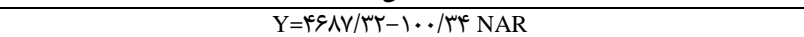 & $\cdot / \cdot$ & $\cdot / \cdot$ \\
\hline$Y=\digamma \Lambda \Delta T / \& q-\Lambda \& / T \Lambda N A R-I T V / \Lambda \& L / S$ & $. / 1 \cdot+\cdot / \cdot 2$ & ./1 \\
\hline 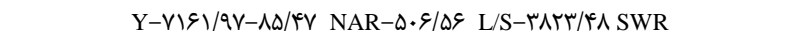 & $.11 \cdot+\cdot 1 \cdot++\cdot \cdot \cdot \cdot 9$ &.$/ 1 \Lambda$ \\
\hline 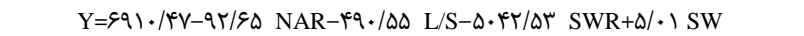 & $. / 1 \cdot+\cdot 1 \cdot t+\cdot / \cdot 8+\cdot 1 \cdot t$ &.$/ \pi$. \\
\hline 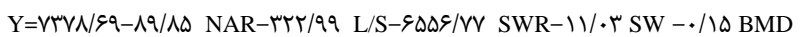 & $. / 1 \cdot+\cdot / \cdot t+\cdot / \cdot c+\cdot / \cdot t+\cdot / \cdot 1$ &.$/ 4$ \\
\hline
\end{tabular}

شد. منفى بودن علامت اين صفت در مدل را مىتوان به دليل

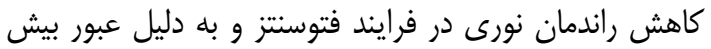

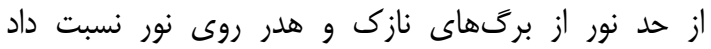

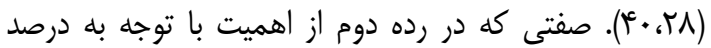

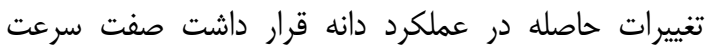

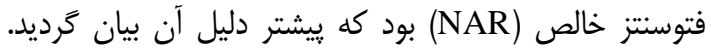

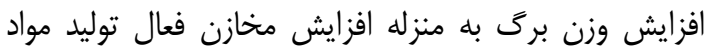

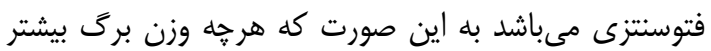

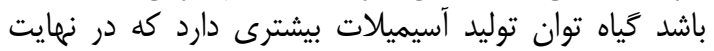

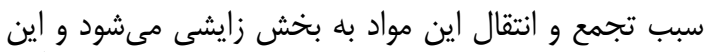

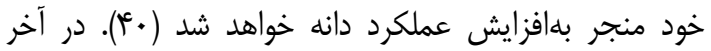
نسبت سطح برى (LAR) توانست تأثير مثبتى بر رون روند

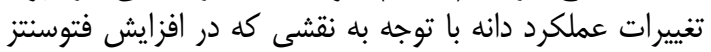

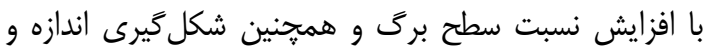

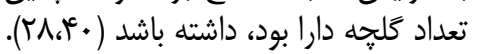

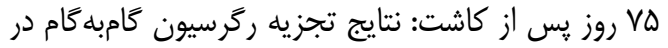

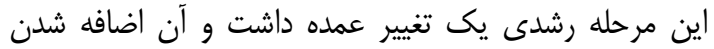

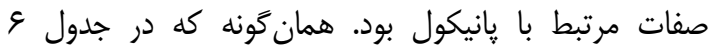

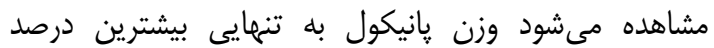

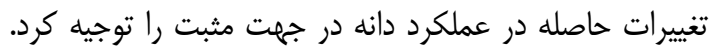

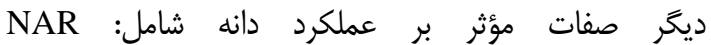

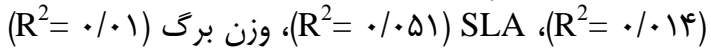

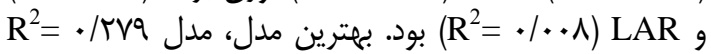

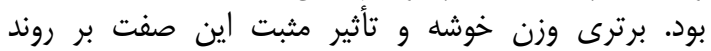

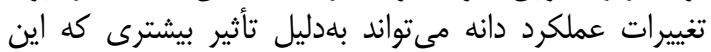

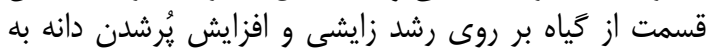

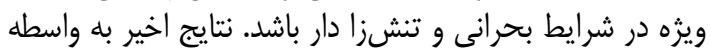

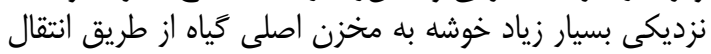

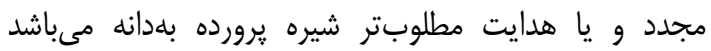

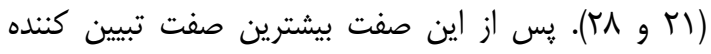

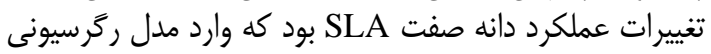




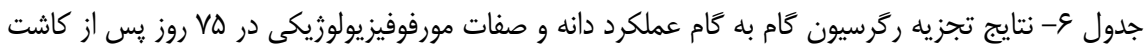
Table 6. Stepwise regression analysis of grain yield and morpho-physiological characteristics after 75 days from

\begin{tabular}{|c|c|c|}
\hline مدل & Partial $R 2$ & Model R2 \\
\hline 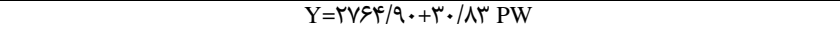 & $\cdot / T$. & $\cdot / T$. \\
\hline$Y=r M Y V / V Q+Y q / 4 Y P W-1 Q I / 9 Q N A R$ & $\cdot / 4 \cdot+\cdot / \cdot 1$ & $. / T \mid$ \\
\hline$Y=F \Delta / F / \Delta F+r N / T$ PW $-r q F / \cdot V$ NAR- $-\Delta \cdot \varphi \cdot 1 / q V$ SLA & $\cdot / 4 \cdot+\cdot / \cdot 1+\cdot / \cdot 0$ & ع ع/. \\
\hline 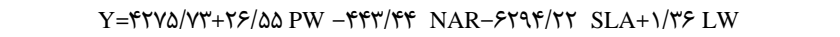 & $\cdot / r \cdot+\cdot / \cdot 1+\cdot / \cdot \Delta+\cdot / \cdot 1$ &.$/ T V$ \\
\hline 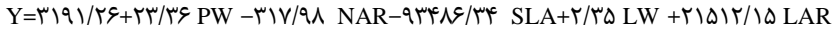 & $\cdot / r \cdot+\cdot / \cdot 1+\cdot / \cdot \Delta+\cdot / \cdot 1+\cdot / \cdot 1$ & $\cdot / T \Lambda$ \\
\hline
\end{tabular}

به رابطه مثبت اين صفت با عملكرد دانه مىتوان نتيجه گرفت

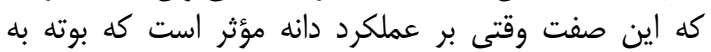

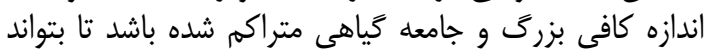

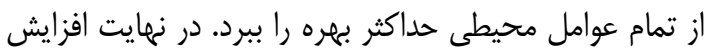

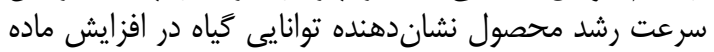

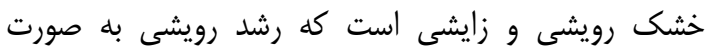

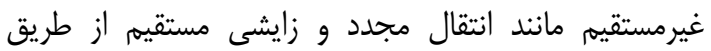

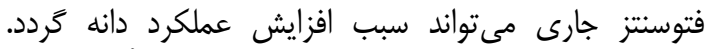

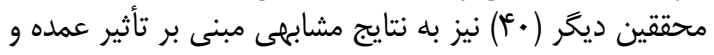

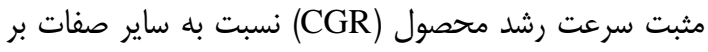
عملكرد دانه در اين مرحله رشدى درل دست يافتند.

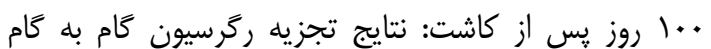

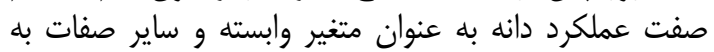

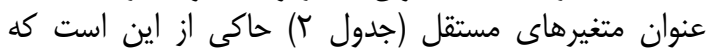

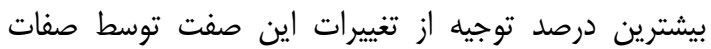

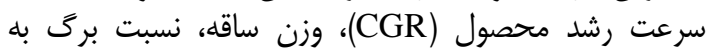

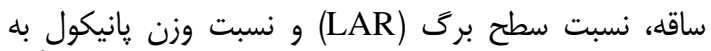

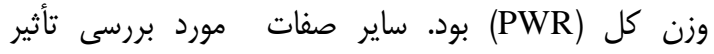

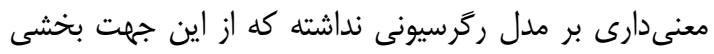

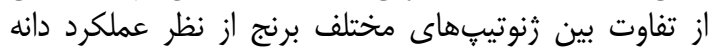

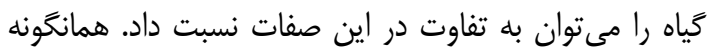

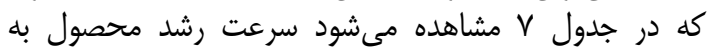

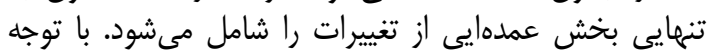

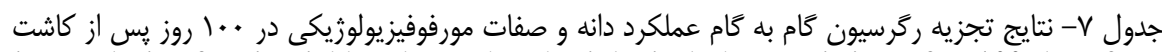
Table 7. Stepwise regression analysis of grain yield and morpho-physiological characteristics after 100 days from

\begin{tabular}{|c|c|c|}
\hline مدل & Partial $R^{2}$ & Model $R^{2}$ \\
\hline$Y=Q r q / F T+r \cdot N / 1 \cdot C G R$ & ד & דז' \\
\hline 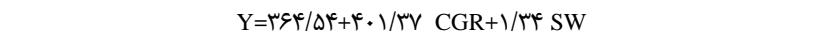 & . &.$/ \mu F$ \\
\hline$Y=|\lambda \digamma N /| \varphi+\varphi q \Delta / \Delta|C G R-| Y \cdot Y / F q * S W-\varphi / \cdot V L / S$ & $. / r+1 \cdot r+\cdot / \cdot 9$ &.$/ \kappa$. \\
\hline$Y=|r \Delta \mu / M \Lambda+\Delta \cdot \cdot / 4 \cdot C G R-r / r \Lambda S W-1 \cdot \Lambda V / \cdot \Delta L / S+| q T \cdot r / q \Psi L A R$ & 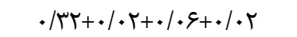 & T4 \\
\hline 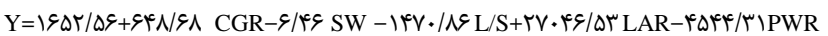 & $. / L+\cdot / \cdot t+\cdot / \cdot t^{2}+\cdot / \cdot t+\cdot / \cdot r$ &.$/ 40$ \\
\hline
\end{tabular}

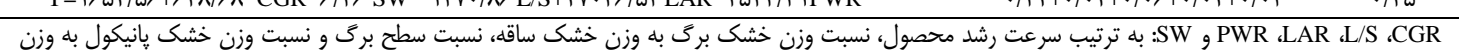

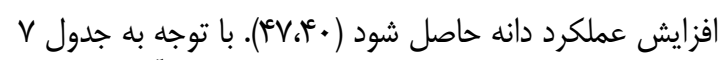

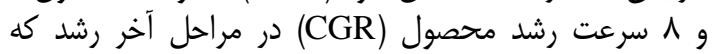

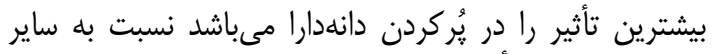

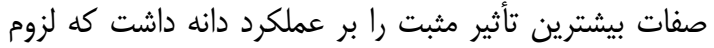

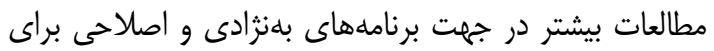

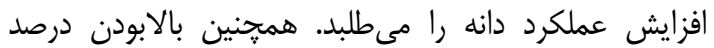

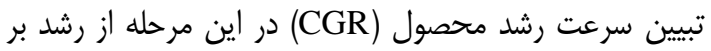

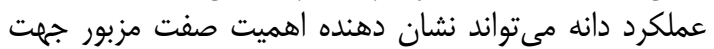

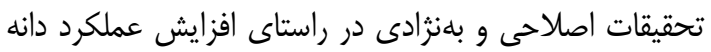

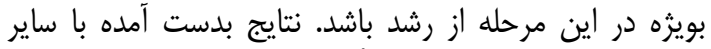

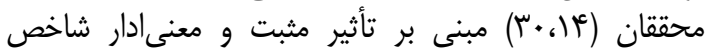

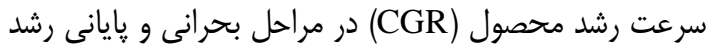

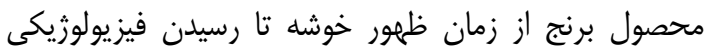

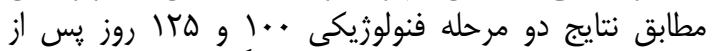

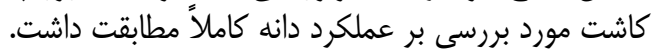

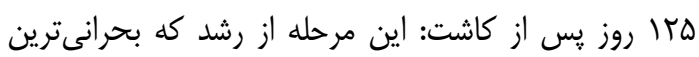

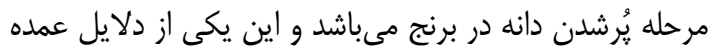

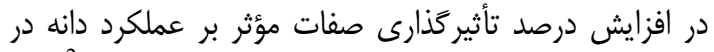

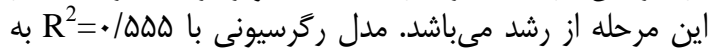

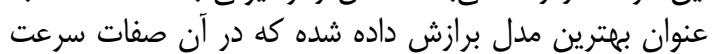

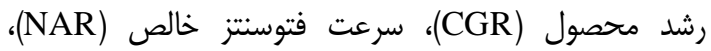

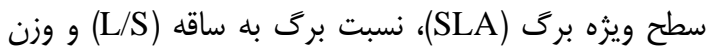

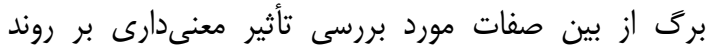

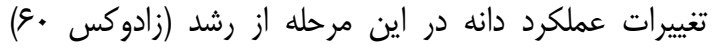

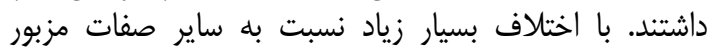

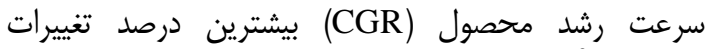

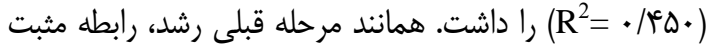

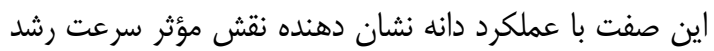

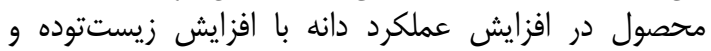

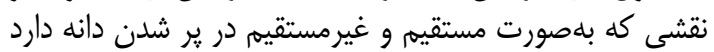

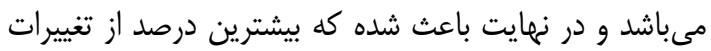




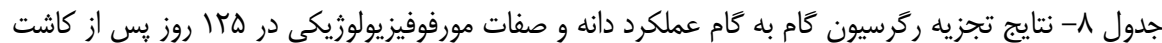
Table 8. Stepwise regression analysis of grain yield and morpho-physiological characteristics after 125 days from planting

\begin{tabular}{|c|c|c|}
\hline مدل & Partial $R^{2}$ & Model $R^{2}$ \\
\hline 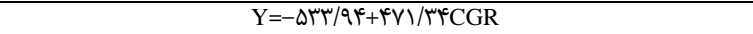 & $\cdot / \uparrow \Delta$ & $\cdot / \uparrow Q$ \\
\hline 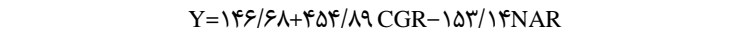 & $\cdot / 4 \Delta+\cdot / \cdot r$ & $\cdot / 4 \wedge$ \\
\hline$Y=q \cdot \wedge / \Delta \Lambda+r \Delta V / / \Lambda C G R-r V \cdot / V V N A R-r q r \varepsilon r / \Delta r S L A$ & $\cdot / 2 \theta+\cdot / \cdot r+\cdot / \cdot t$ & $\cdot / \Delta \cdot$ \\
\hline 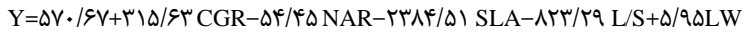 & $\cdot / k g+\cdot / \cdot r+\cdot / \cdot t+\cdot / \cdot t+\cdot / \cdot t$ & $\cdot / \Delta \omega$ \\
\hline
\end{tabular}

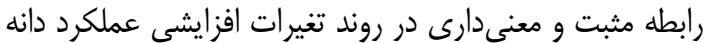

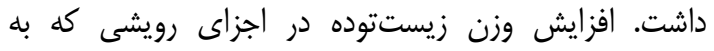

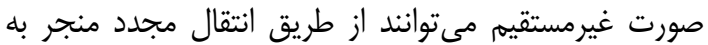

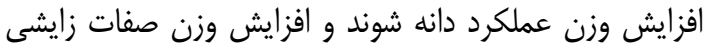

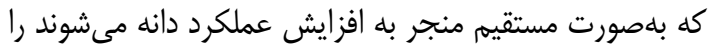

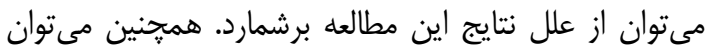

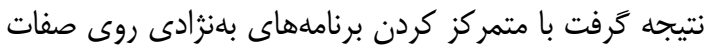

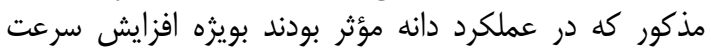

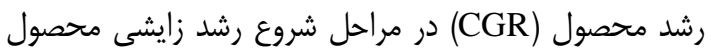

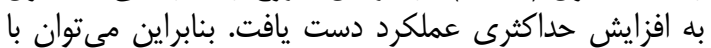

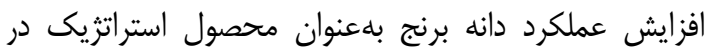

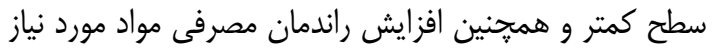

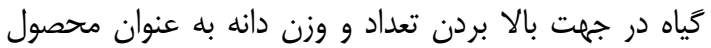

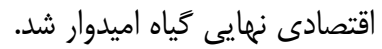

نتايج حاصل از بررسىهاى رگرسيونى نشان داد كه با

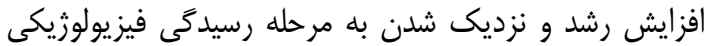

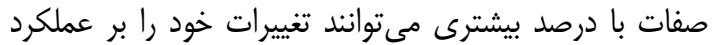

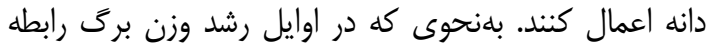

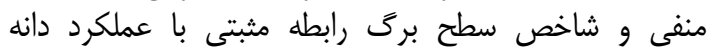

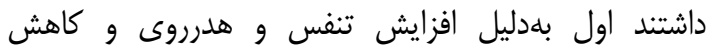
راندمان مصرفى انرزى و دوم به دليل افزايش فت فتوسنتز و و مواد

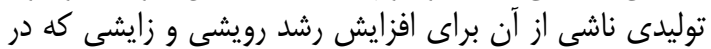

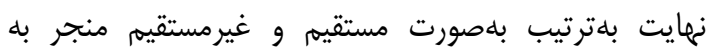

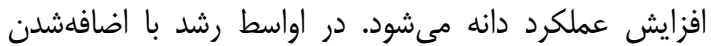

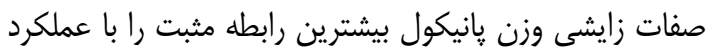

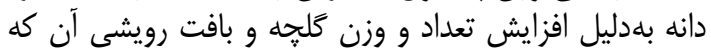

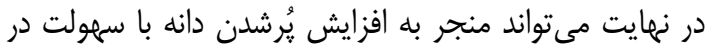

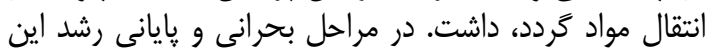

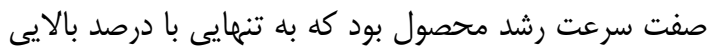

1. Akbari, G.h., R.A. Salehi Zarkhuni, M. Yvsfy-Rad, M. Nasiri, C. Motaghi and W.A. Ltfy-Fr. 2007. Evaluation of some morphological characteristics affecting the performance and yield components of rice genotypes. Journal of Agricultural Sciences, 3(2): 137-130 (In Persian).

2. Arfani, A. and M. Nasiri. 2001. Evaluation of some morphological and physiological characteristics affecting rice yield. Publications Rice Research Institute-Department of Mazandaran, 43 pp (In Persian).

3. Baradaran, R., E. Majidi, F. Darvish and M. Azizi. 2007. Study of Correlation Relationships and Path Coefficient Analysis Between Yield and Yield Components in Rapeseed (Brassica napus L.). Journal of Agricultural Sciences, 12(4): 811-818 (In Persian).

4. Dawari, N.H. and O.P. Luthra. 1991. Character association studies under high and low environments in wheat (Triticum aestivum L.). Indian. Journal of Agricalture Research, 25: 68-72.

5. Durand, M., B. Porcheron, N. Hennion, L. Maurousset, R. Lemoine and N. pourtau. 2016. Water Deficit Enhances C Export to the Roots in Arabidopsis thaliana Plants with Contribution of Sucrose Transporters in Both Shoot and Roots. Plant Physiology, 170(1): 1460-1479.

6. Dutta, R.K., M.A. Baset Mia and S. Khanam. 2002. Plant architecture and growth characteristics of fine grain and aromatic rices and their relation with grain yield. Bangladesh Crop Physiology, 32: 95-102.

7. Egli, D.B. 1998. Alternation in plant growth and dry matter distribution in soybean. Agronomy Journal. 80: $86-90$.

8. Farhodi, R., M. Kuchk-Pur and A.R. Safahani Langroodi. 2007. Evaluation of salt tolerance mechanisms in three varieties. Proceedings of the First Symposium on canola and canola oil. Islamic Azad University Branch, 300 pp (In Persian).

9. Farshadfar, E., G. Galiba, B. Kozsegi and J. Sutka. 1993. Some aspects of the genetic analysis of drought tolerance in wheat. Cereal Research Communications, 21: 323-330.

10. Gent, M.P.N. 1995. Canopy light, gas exchenge and biomass in reduced height of winter wheat isolines of winter wheat crop. Crop Science, 38: 36-42.

11. Gravois, K.A. and R.S. Helms. 1992. Path analysis of rice yield and yield components as affected by seedling growth rate. Agronomy Journal, 84: 1-4.

12. Hashemi Dezfooli, A., A. Kochaci and M. Bnaean. 1995. Increased crop yield. Publications University of Mashhad, 243 p (In Persian).

13. Hashemi, Z.A., A.A. Gol-Prvr and M. Rasoli. 2008. Analysis of correlation, regression and causality performance and yield components of rapeseed (Brassica napus L.). The new findings agriculture, 2(4): 419-412 (In Persian).

14. Horie, T., H. Yoshida, T. Shiraiwa, H. Nakagawa, E. Kuroda, T. sasaki, M. Hagiwara, T. Kobata, M. Ohnishi and K. Kobayashi. 2003. Analysis of benotype by environment interaction in yield formation processes of rice grown under a wide environmental range in Asia. 10. Asia Rice Network (ARICENET) research and preliminary results. Japanise Journal of Crop Science, 72(2): 88-89 (In Japanese). 
15. Horton, P. 2000. Prospects for crop improvement trought the genetic manipulation of photosynthesis morphological and biochemical aspect of light capture. Journal of experimental Botany, 51: 475-485.

16. Kobata, T., M. Sugware and S. Tcx Akata. 2000. Shading during the early grain filling period not affect potential grain dry matter increase in rice. Agronomy Journal, 92: 411-417.

17. Kochaci, A. and M. Bnaean. 1994. Physiology crop yield. Publications University of Mashhad, 380 pp (In Persian).

18. Khajepour, M.R. 2008. Principals and Essentials of crop production. Jihad-University Press. Isfahan University of Technology, $388 \mathrm{pp}$ (In Persian).

19. Khodabande, N. 1998. Cereals. Tehran University Press. 537 pp (In Persian).

20. Kush, G.S. 1993. Breeding rice for sustainable agriculture system. Crop Science Society of America, 1: 189-199.

21. Limouchi, K., S.A. Siadat, A. Gilani. 2013. Sowing dates effect on yield and growth indexes of rice cultivars in northern Khozestan. Iranian society of Agronomy and plant breeding sciences, 6(2): 167-184.

22. Mahdavi, F. 2004. Study physiological and morphological indices of growth in new and old varieties of rice. Mazandaran University master's thesis, $131 \mathrm{p}$ (In Persian).

23. Mahdavi, F., M.A. Ishmael, A. Falah and H. Pirdashti. 2005. The study morphological, physiological indices, yield and yield components in Landrace and Improved Rice (Oryza sativa L.). Iranian Journal of Crop Sciences, 7(4): 297-280 (In Persian).

24. Matsou, T., K. Kumazawa, R. Ishii, K. Ishihara, and H. Hirata. 1995. Science of the rice plant. Food and Agriculture Policy Research Center of Japan, Volume2: Physiology.

25. Mohammadi, S., M. Nahavi and A. Mohadasi. 2015. Effects of intermittent irrigation at different growth stages on yield and yield components of rice cultivars. Journal of Research and Development (Agriculture), 2(107): 114-108 (In Persian).

26. Mohammadnia, S., A. Asghari, O. Sofalian, H, Mohammaddoust-Chamanabad, R. Karimizadeh, and A. Shokouhian. 2017. Evaluation of Durum Wheat Lines using Drought Stress Indices. Journal of Crop Breeding, 8(20): 11-23 (In Persian).

27. Mohd-Zain, N.A. and M. Razi-Ismail. 2016. Effects of potassium rates and types on growth, leaf gas exchange andbiochemical changes in rice (Oryza sativa) planted under cyclic water stress. Agricultural Water Management, 164(1): 83-90.

28. Mosavy, S.A., M.R. Khaledian, A. Ashrafzadeh and P. Shahinrokhsar. 2016. Effects of limited irrigation on yield and water productivity increasing of three soybean genotypes in Rasht region. Journal of water research agriculture, 29(4): 433-446.

29. Murchie, E. H., Y. Z. Chen, S. Hubbart, S. Peng and P. Horton. 1999. Interactions between Senescence and Leaf Orientation Determine in Situ Patterns of Photosynthesis and Photoinhibition in Field-Grown Rice. Plant Physiology, 119: 553-563.

30. Murchi, E., Y. Jian chang, H. Stella, H. Peter and P. Shaobing. 2002. Are there associations between grain filling rate and photosynthesis in the flag leaves of field grown rice? Journal of Experimental Botany, 53(378): 2217-2224.

31. Nasiri. M., M. Bahrami and S. Hosseini. 2002. The introduction of new rice varieties with desirable qualities. Publishing your research institution rice, $22 \mathrm{pp}$ (In Persian).

32. Nourbkhshyan, J. and A. Rezai. 1998. curve and growth rate of rice in the region lordagan. Congress Crop Iran, Karaj, Seed and Plant Improvement Institute, September 11-13. pp. 621-620 (In Persian).

33. Nourbkhshyan, J. and A. Rezai. 2008. Correlation between quantitative traits of grain yield rice through path analysis. Iranian Journal of Crop Sciences, 4(1): 25-35 (In Persian).

34. Peng, S. and D. Senadhira. 2003. Genetic enhancement of rice yields. Crop Science, 45: 1238-1246.

35. Pirdashti, H. 1998. Evaluation of the recently opened, re-planting on the transfer of nitrogen and growth indices, yield rice varieties. Agriculture Master's thesis. Tarbiat Modares University. Agriculture is composed of eight, $158 \mathrm{p}$ (In Persian).

36. Rahimian, H., A. Kochaci and A. Zand. 1998. Evolution and adaptation and crop yield. Publishing publishing agricultural education, $241 \mathrm{p}$.

37. Sahara T., T. Takahashi, T. Kayaba and S. Tsunada. 1992. A new strategy for increasing plant productivy in rice. International Rice Common News, 41:1-4.

38. Saleh, J., N. Alh-Njfy, S.H. Avestan. 2015. Effect of silicon on growth, chemical composition and properties Fyzvlvzhyky some rice in saline conditions. Journal of Science and Technology of Agriculture and Natural Resources, 19(72): 240-229 (In Persian).

39. Sheikh, F. A., A.G. Rather and S.A. Wani. 1999. Genetic variability and inter-relationship in Toria (Brassica campestris L. var. Toria). Advances in Plant Sciences, 12(1): 139-143.

40. Tan, X., D. Shao, H. Liu, F. Yang, C. Xiao and H. Yang. 2013. Effects of alternate wetting and drying irrigation on percolation and nitrogen leaching in paddy fields. Paddy Water Environ, 11: 1-15.

41. Tavoosi, M., A. Naderi and G.H.A. Ltfly-Ynh. 2015. Evaluation of wheat genotypes reaction to cold stress at heading stage using physiological indices, yield and yield components. Field Crop Science Iran, 46(1): $113-105$.

42. Wells, R., J.V. Borton and T.C. Kilen. 1993. Soybean growth and interception response to differing leaf and stem morphology. Crop Science, 33: 520-524.

43. Yadava, M.S. and O.P. Singh. 1988. Effect of plant growth characters on yield of indian rice cultivars. Indian Journal of Botany, 11: 74-83.

44. Yamauchia, M. 1994. Physiological bases of height yield potential in F1 rice hybrids. Hybrid Rice Technology. 3rd ed., International Rice Research Institute Press, 324 pp.

45. Yang, J., S. Peng, Z. Zhang, Z. Wang, R.M. Visperas and Q. Zhu. 2002. Grain and dry matter yields and partitioning of assimilates in Japonica/Indica hybrid rice. Crop Science, 42: 766-772.

46. Yap, T.C. and B.L. Harvey. 1972. Inheritance of yield components and morpho-physiological traits in barley (Hordeum vulgare L.). Crop Science, 12: 283-286.

47. Zali, H., T. Hasanloo, O. Sofalian, A. Asghari and M. Zeinalabedini. 2017. Appropriate Strategies for Selection of Drought Tolerant Genotypes in Canola. Journal of Crop Breeding, 8(20): 77-90. 


\title{
Study of Regression Analysis of Rice Physiological Indices in Different Growth Stages under Khuzestan Condition
}

\section{Kaveh Limouchi ${ }^{1}$, Mehrdad Yarnia ${ }^{2}$, Ataollah Siyadat $^{3}$, Varahram Rashidi $^{4}$ and Abdolali Guilani}

\author{
1 and 4- Ph.D. Student and Assistant Professor, Department of Agronomy, Faculty of Agriculture, Tabriz Branch, \\ Islamic Azad University \\ 2- Professor, Department of Agronomy, Faculty of Agriculture, Tabriz Branch, Islamic Azad University \\ (Corresponding author: yarnia@iaut.ac.ir) \\ 3- Professor, University of Agricultural and Natural Resources of Ramin, Ahwaz. \\ 5- Assistant Professor of Seed and Plant Improvement Research Department, Khozestan Agricultural and Natural \\ Resources Research Center, AREEO, Ahvaz. \\ Received: February 14, 2017 \\ Accepted: August 13, 2017
}

\begin{abstract}
This study aimed to investigate the relationship between morphophysiological and phenology traits with grain yield to determine the best indices of direct and indirect selection in the field of genetic improvement of aerobic rice grain yield in Khuzestan province with 12 aerobic rice genotypes. The traits such as include the grain yield as the dependent variable and total dry matter, panicle weight, stem weight, LAI, CGR, NAR, LAR, SLA, ALW, LWR, L/S, SWR, PWR, LAD and BMD as independent variables, which were analyzed by stepwise regression at different growth stages to determine their effect on grain yield. The results showed that on 25 days after planting traits of L/S, SLA, LAI and leaf weight, on 50 days after planting traits of NAR, L/S, SWR, stem weight and BMD, on 75 days after planting traits of NAR, SLA, stem and LAR, on 100 days after planting traits of CGR, stem, L/S, LAR and PWR and on 125 days after planting traits of CGR, NAR, SLA, L/S and leaf affected the grain yield. In order from the beginning to the end of the growth, traits of L/S, NAR, panicle weight, CGR and CGR had caused the greatest changes in the grain yield. According to the critical period of growth, CGR was the most effective trait on the grain yield because of allocation of more assimilates in the reproductive growth instead of vegetative growth during grain filling period, thus it can be an important objective in the breeding researches.
\end{abstract}

Keywords: CGR, Growth stages, Morphology, Physiology, Stepwise regression 